\title{
Measurement by FIB on the ISS: Two Emissions of Solar Neutrons Detected?
}

\author{
Y. Muraki, ${ }^{1}$ K. Koga, ${ }^{2}$ T. Goka, ${ }^{2,3}$ H. Matsumoto, ${ }^{2}$ T. Obara, ${ }^{2,4}$ O. Okudaira, ${ }^{2}$ \\ S. Shibata, ${ }^{5}$ and T. Yamamoto ${ }^{6}$
}

${ }^{1}$ Solar-Terrestrial Environment Laboratory, Nagoya University, Nagoya 464-8601, Japan

${ }^{2}$ Tsukuba Space Center, JAXA, Tsukuba 305-8505, Japan

${ }^{3}$ Department of Physics, Tokyo Metropolitan University, Tokyo 192-0397, Japan

${ }^{4}$ Planetary Plasma and Atmospheric Research Center, Tohoku University, Sendai 980-8578, Japan

${ }^{5}$ Department of Information Science, Chubu University, Kasugai 487-8501, Japan

${ }^{6}$ Department of Physics, Konan University, Kobe 658-8501, Japan

Correspondence should be addressed to Y. Muraki, muraki@stelab.nagoya-u.ac.jp

Received 15 August 2012; Revised 14 October 2012; Accepted 29 November 2012

Academic Editor: Karel Kudela

Copyright ( $\odot 2012$ Y. Muraki et al. This is an open access article distributed under the Creative Commons Attribution License, which permits unrestricted use, distribution, and reproduction in any medium, provided the original work is properly cited.

\begin{abstract}
A new type of solar neutron detector (FIB) was launched on board the Space Shuttle Endeavour on July 16, 2009, and began collecting data at the International Space Station (ISS) on August 25, 2009. This paper summarizes the three years of observations obtained by the solar neutron detector FIB until the end of July 2012. The solar neutron detector FIB can determine both the energy and arrival direction of neutrons. We measured the energy spectra of background neutrons over the South Atlantic Anomaly (SAA) region and elsewhere and found the typical trigger rates to be 20 and 0.22 counts/sec, respectively. It is possible to identify solar neutrons to within a level of 0.028 counts/sec, provided that directional information is applied. Solar neutrons were possibly observed in association with the M-class solar flares that occurred on March 7 (M3.7) and June 7 (M2.5) of 2011. This marked the first time that neutrons had been observed in M-class solar flares. A possible interpretation of the production process is provided.
\end{abstract}

\section{Introduction: A Brief History of Solar Neutron Detection}

High-energy protons coming from the Sun on February 28 and March 3, 1942 were first discovered by Forbush and published in 1946 [1]. In 1951,Biermann et al. had predicted the potential discovery of solar neutrons on Earth [2]. Neutrons are produced when the accelerated ions strike the solar surface. However, solar neutrons were actually detected 29 years after this prediction. A clear signal of gamma rays and neutrons was detected in association with a large solar flare on June 21, 1980, with an X-ray intensity of X2.5, by the Gamma Ray Spectrometer composed of the NaI and CsI detectors on board the Solar Maximum Mission (SMM) satellite $[3,4]$. Figure 1 shows the results. The first peak corresponds to the gamma-ray signal, while the second was induced by the neutron signal. Because neutrons cannot travel from the Sun to Earth at the speed of light, their arrival time distribution is associated with a time delay from the speed of light, even when simultaneously released from the Sun. For the time distribution presented in Figure 1, if the same departure time for neutrons is set, a neutron energy spectrum is obtained, which assumed an impulsive production of neutrons on the Sun. The spectrum can be expressed by a power law: $E_{\mathrm{n}}^{-\gamma} \mathrm{d} E_{\mathrm{n}}$ with $\gamma=3.5 \pm 0.1[3,4]$.

Two years later, on June 3,1982, the SMM satellite again detected a neutron signal [5]. However, neutron monitors located on the ground have successively detected neutron signals in association with a large X8.2 solar flare $[6,7]$, which shed new light on the production time of neutrons in the solar atmosphere. One component involved in the data cannot be explained by an impulsive production mechanism alone. Only two solar neutron events had been accumulated until solar cycle 21 , and it was too early to judge the production time of neutrons in the solar atmosphere, namely, whether high-energy neutrons are produced impulsively 


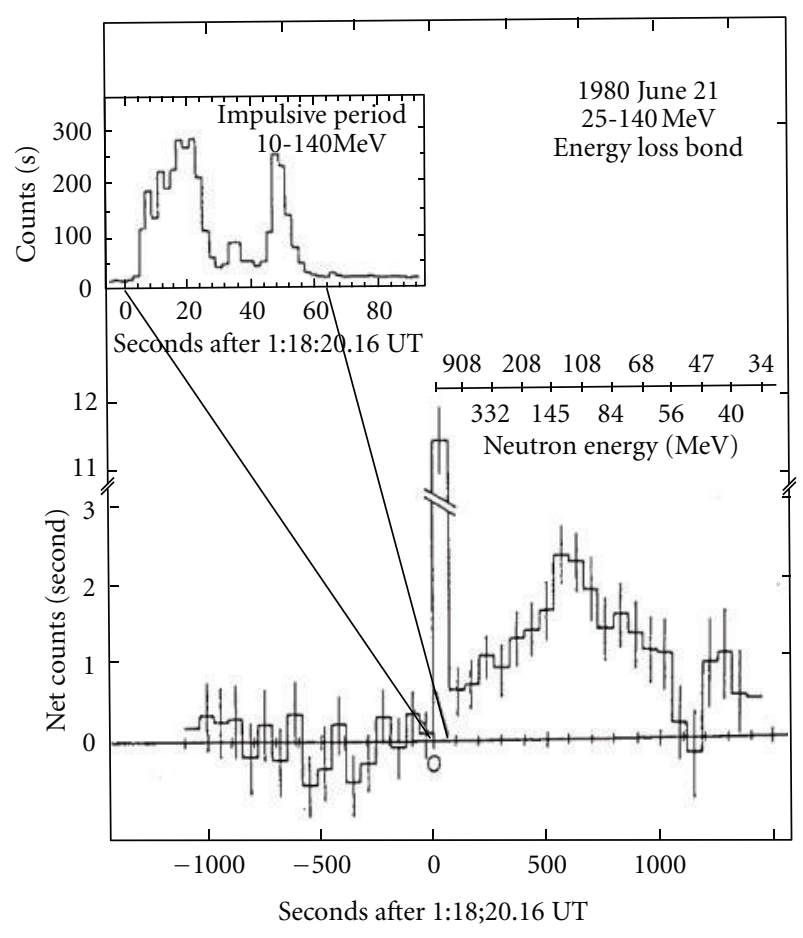

Figure 1: Solar neutrons detected on June 21, 1980. Gamma-rays accounted for the first peak; neutrons accounted for the second. The original picture was prepared by the authours of $[3,4]$.

or gradually. Both scenarios were possible for the same event and this would be a great challenge for solar physicists.

To identify the production time of neutrons at the solar surface in the solar cycle 22, new detectors capable of measuring the energy of neutrons were expected. Therefore a new type of solar neutron detector-the solar neutron telescope (SONTEL) - was designed, based on the plastic scintillator. SONTEL can measure the energy and direction of neutrons using the charge exchange process of neutrons into protons $[8,9]$. Therefore SONTEL can determine the flight time from the Sun to Earth. Of course, conventional Simpson-type neutron monitors were also operated [10-13]. At the same time, the possibility of launching a new type of solar neutron detector into space was considered (the idea was proposed in a symposium at Nagoya University held on April 17 and July, 1989. Also presented in the symposiums organized by JSUP of NASDA). To resolve the mystery regarding the production time of neutrons, it is inevitable to have a new type of detector capable of measuring the energy of neutrons in space. We could then identify when those neutrons left the Sun.

An attempt to measure the energy of neutrons was found in a paper dated around 1985 [14]. Scintillator bars composed of two layers were equipped (in $x$ and $y$ directions) and the device was circulated over the Southern hemisphere by a Racoon balloon flight from Alice Springs, Australia to detect solar neutrons within the energy range of 20 to $150 \mathrm{MeV}$, but detected no signals. Almost the same year 1985, another new instrument was proposed, capable of unambiguously determining the energy and direction of incident neutrons using a technique, the double Compton scattering method [15]. The detector was named SONTRAC. Independent from these activities, most of which were developed in the USA, in Japan, a detector comprising a mass of the scintillation fiber is proposed to detect antideuterium in space and neutron and anti-neutron oscillation in the space between the Sun and Earth. In 1991, we proposed a new type of detector for the Japanese Experimental Module (JEM) of the International Space Station (ISS) $[16,17]$.

In April 1991 and August 1991, a large gamma-ray satellite CGRO and a solar satellite Yohkoh were launched. An image of the Sun using neutron signals was successfully drawn, using the Compton scattering function of the COMPTEL detector $[18,19]$ and beautiful photographs of solar flares were taken by using the soft and hard X-ray telescope of Yohkoh satellite. They have left very important archives on the solar activities [20].

During the solar cycle 22, several new discoveries involving solar neutrons were made, based not only on many ground level detectors but also a few that were space-borne. Consequently, more solar neutron events were accumulated, including one highlight, the discovery of an extremely strong signal of neutrons in association with the large X9.3 solar flare on May 24, 1990 [21, 22]. The signal was the strongest ever observed by the neutron monitor. In association with this flare, two Soviet satellites, GRANAT/PHEBUS [23] and GAMMA-1 [24, 25] successfully captured very impulsive high-energy gamma rays starting at 20:48 UT. One minute later (20:49 UT), strong neutron signals were detected by many neutron monitors located throughout the North American continent [26, 27]. Subsequently, from around 21:00 UT, Ground Level Enhancement (GLE) was observed, induced by high-energy protons. The key knowledge obtained by the event on May 24, 1990, reviewing some 20 years after the discovery, may be the sudden increase in the ratio between 70 and $95 \mathrm{MeV}$ gamma rays and 4$7 \mathrm{MeV}$ nuclear gamma rays 3 minutes later. Chupp and Ryan summarized that the change in ratio may have been induced by accelerated protons to several hundred $\mathrm{MeV}[28,29]$. It is worth noting that to detect high-energy neutrons at ground level, conventional neutron monitors were not only used to detect solar neutrons in the large solar flare on May 24, 1990, but also in the X9.4 flare on March 22, 1991 [30].

The subsequent scope of remarkable events from solar cycle 22 may also include the detections of high-energy gamma rays and neutrons in association with the six extremely powerful solar flares with X12 observed during June 1 and 15, 1991. Solar neutrons were detected in association with two large solar flares on June 4 and 6, 1991, respectively, using two kinds of solar neutron detectors located on Mt. Norikura: the solar neutron telescope $[31,32]$ and the neutron monitor [33]. Via simultaneous observations with the neutron monitor and the neutron telescope, the capability of the new solar neutron telescope was demonstrated.

It should be mentioned that in the solar flare on June 4, 1991, the BATSE [34] and OSSE [35] detectors on board the CGRO satellite observed the long-standing emission of gamma rays with a decay time of 330 seconds after 
a sharp impulse signal. OSSE observed a neutron capture line $(2.223 \mathrm{MeV})$ and a carbon de-excitation line $(4.44 \mathrm{MeV})$ that continued for three hours. High-energy gamma rays were detected by the EGRET detector with energies of 50 to $100 \mathrm{MeV}$ and $>150 \mathrm{MeV}$ for the flare events on June 4, 6, 9, and 11. Moreover, in the flare event on June 11, a particularly long-lasting emission of high-energy gamma rays was recorded, lasting 10 hours [36].

Many arguments concerning the long-lasting gamma rays emerged at the time, namely whether they were induced by the continuous acceleration process of protons (such as in the shock acceleration model [37]) or by protons trapped in the magnetic loop and precipitating on the solar surface $[38,39]$. The impulsive production mechanism of neutrons on the solar surface was attributable to the reconnection process of magnetic loops [40-43] or the DC acceleration mechanism [44], while long-lasting emissions of gamma rays may be closely related to the shock acceleration process. The question of whether the long-lasting high-energy gammaray emission is attributable to the continuous acceleration of the protons above $300 \mathrm{MeV}$ [29], or the injection of flare accelerated particles into a large coronal loop with release at the mirror points of the loop where the gamma rays are produced, is very interesting $[38,39]$, the final answer to which will hopefully be obtained in solar cycle 24 .

During the solar flare event on September 7, 2005, solar neutron telescopes located on Mt. Sierra Negra in Mexico (at $4780 \mathrm{~m}$ ) and Mt. Chacaltaya in Bolivia (at 5,250 m) both observed a clear solar neutron signal [45], which was also recorded by three different counters located in the Northern and Southern Hemispheres. This made it possible to compare the detection efficiency of a solar neutron telescope with that of a conventional neutron monitor. The detection efficiency ratios were found to be 1 and 0.7 , for the neutron monitor and neutron telescope respectively, pertaining to the same area of both detectors. Since the solar neutron telescope cuts low-energy neutrons of less than 30 or $40 \mathrm{MeV}$, its detection efficiency is also lower than that of the neutron monitor [46]. The neutron monitor is highly sensitive to neutrons with energy exceeding about $10 \mathrm{MeV}$ $[12,13]$. It is worth noting here that the data suggests the involvement of neutrons produced by both the impulsive and gradual phases [45].

The FERMI-LAT satellite also recently observed two gamma-ray events in association with M-class solar flares on March 7 and June 7, 2011 [47]. Again a long duration component lasting more than 14 hours was observed and the continuous emission of $\mathrm{GeV}$ gamma rays from the Sun was detected. This mechanism may indicate a different mechanism in the gamma-ray production process in addition to that responsible for the impulsive production of gamma rays, which is discussed in the final part of this paper. An effective summary on solar neutron research has been recently published in a book, which also contains more detailed bibliography [48].

The aim of this paper is to present new results using the FIB detector on board the ISS. Actually Section 2 introduces details of the new solar neutron telescope FIB detector, followed by the neutron observation results on the ISS in
Section 3. Section 4 covers the solar neutron events observed using the new detector in association with the M-class solar flares on March 7, 2011 (M3.7), and on June 7, 2011 (M2.5). Section 5 discusses our results compared to other observations, and Section 6 summarizes the results.

\section{New Solar Neutron Detector FIB on the ISS}

2.1. SEDA-AP-FIB Detector. The new solar neutron telescope has been designed as a component of SEDA-AP. A detector for Space Environment Data Acquisition equipmentAttached Payload (SEDA-AP) was originally proposed to measure radiation levels at the International Space Station (ISS) in 1991 [16, 17]. In 2001, an actual Flight Module (FM) was ready to be deployed, but an accident involving the Space Shuttle resulted in the FM being stored in a special clean room for eight years until it could finally be launched.

SEDA-AP was designed as one of the detectors on board the Japan Exposure Module (JEM). This equipment not only comprises a neutron detector but also various other detectors, such as charged particle detectors, a plasma detector, an atomic oxygen monitor, and electronic device evaluation equipment. The system even includes a microparticle capture detector.

The neutron detector consists of two parts: a conventional Bonner Ball Detector (BBD) and a FIBer detector (FIB). The name FIB is so-called because the main part of the sensor is comprised by the mass of the scintillation fiber. The BBD measures low-energy neutrons; the FIB measures highenergy neutrons. Technical details can be found on the JAXA website (http://kibo.jaxa.jp/en/experiment/ef/seda-ap/).

The neutron detector can be extended $1 \mathrm{~m}$ from the main frame via a mast to reduce the background neutrons coming from the vessel of the SEDA-AP. The system has a 220-watt power supply and a total weight of $450 \mathrm{~kg}$. The FIB was launched by the Space Shuttle Endeavour on July 16, 2009, and began taking measurements at the ISS on August 25, 2009. Since then, the detectors have been working problemfree. Although the official mission lifetime was estimated as three years, given the importance of the measurements, it would be highly desirable to extend this period to cover at least one solar cycle of 11 years, provided that the system continues to operate.

2.2. The Experimental Purposes. This experiment has three main scientific goals as follows.

(1) Accurate measurements of radiation levels in the ISS environment [49-51].

(2) Rapid prediction of the imminent arrival of numerous charged particles from the Sun by monitoring $\mathrm{GeV}$ GLE particles for the flares of the western part of the solar surface (space weather forecast). However for the flares of the eastern part of the solar surface, the amount of emitted high energy particles may be estimated by observing neutrons.

(3) Identification of the production time of neutrons induced by the accelerated protons above the solar 
surface. We wish to know when and how highenergy particles are produced over the solar surface. When high-energy charged particles arrive at Earth and are detected, important information may be lost concerning the production time at the Sun. To understand the acceleration mechanism of charged particles at the Sun, it is necessary to compare the data of neutrons and gamma rays with images taken by a soft X-ray telescope [52], RHESSI, and/or the UV telescope launched on the Solar Dynamical Observatory $[53,54]$.

To determine the neutron production time at the Sun, it is necessary to employ a neutron detector capable of measuring the energy of neutrons. Currently, no such detector has been used in space other than an FIB detector, although the ground-based Solar Neutron Telescopes (SONTEL) have been operating for a number of years [55-58]. Accordingly, the FIB detector installed in SEDA-AP may provide a crucial data measuring neutron energy in space in the solar cycle 24 .

2.3. Sensor Design, Detection Efficiency, and Trigger. To achieve the scientific goals listed earlier, a fine-grated neutron detector FIB has been designed, consisting of a plastic scintillator with 32 layers (sheets) and dimensions of $3 \mathrm{~mm}$ (height) $\times 96 \mathrm{~mm}$ (width) $\times 96 \mathrm{~mm}$ (length). Sixteen stacks of scintillation bars are used per layer, with each bar having dimensions of $3 \mathrm{~mm}$ (height) $\times 6 \mathrm{~mm}$ (width) $\times 96 \mathrm{~mm}$ (length). Each layer is located along the $x$-axis and $y$-axis alternatively, forming a stratified block and an optical fiber is coupled to the end of each bar to collect photons produced in the scintillator. These photons are then sent to a 256channel multi-anode-photomultiplier (Hamamatsu H414020). Figure 2 shows a schematic image of the FIB detector, which measures the tracks of recoil protons produced by incident neutrons and determines neutron energy using the range method.

It can also identify the direction of neutron incidence. Neutrons and protons are discriminated by an anticoincidence system consisting of six scintillator plates surrounding the FIB sensor in a cubic arrangement. To measure the total radiation dose at the ISS, we actually collect neutron data obtained over the South Atlantic Anomaly (SAA) region. The maximum count rate of the anti-coincidence system for the SAA region is 60,000 counts per second, and it works.

The cubic-shaped sensor used for neutron detection has sides measuring $10 \mathrm{~cm}$ and maximum kinetic energy of about $120 \mathrm{MeV}$. As shown in Figure 3, the sensor is monitored from two directions by two multi-anode photomultipliers (PMT1 and PMT2), meaning the arrival direction of the tracks can be identified. To determine the arrival direction of neutrons, protons must penetrate at least four sensor layers, each of which consists of plastic bars 3-mm thick. Consequently, the lowest neutron energy that can be measured is $35 \mathrm{MeV}$.

A trigger signal is produced by dynode signals from the PMTs (it is set at $\gtrsim 30 \mathrm{MeV}$ proton equivalent). When the dynode signals from both PMTs exceed a certain threshold, a trigger signal is produced. When the trigger rate is less than

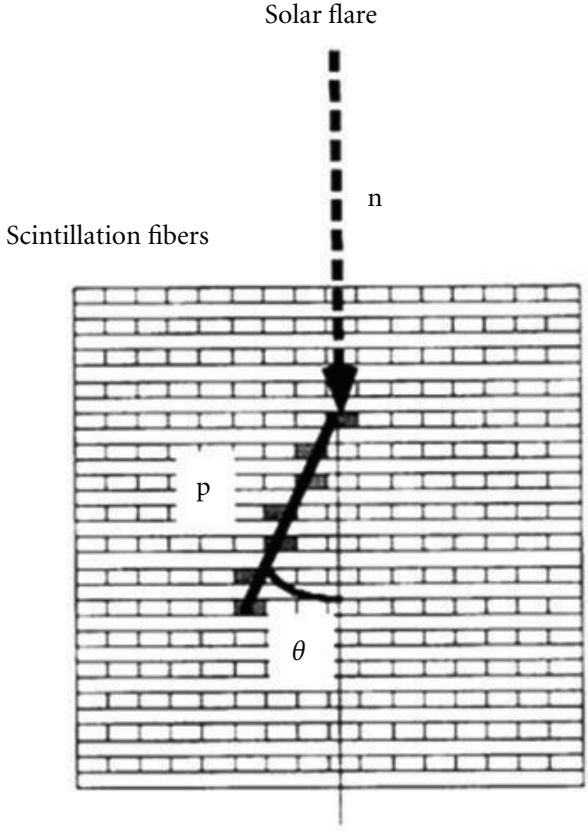

Figure 2: Schematic view of the FIB detector's sensor. One layer consists of 16 plastic scintillator bars with dimensions of $3 \mathrm{~mm}$ (height) $\times 6 \mathrm{~mm}$ (width) $\times 96 \mathrm{~mm}$ (length). The direction of the Sun is identified by tracking two layers of the scintillator in both $x$ and $y$ directions; the energy of neutrons is measured by the range of protons.

2 counts/sec, all ADC values for each channel are recorded in memory. The analog memory can handle all 512 channels of both PMTs. When the trigger rate exceeds 2 counts/sec, only the on-off signal (1 or 0 ) of each channel is recorded. When it exceeds 15 counts/sec, only the total output signal of the dynode is recorded. The technical details can be found elsewhere [59-62].

2.4. Detection Efficiency. We now discuss the neutron detection efficiency of the sensor. Because of the cubic-shaped detector has sides measuring $10 \mathrm{~cm}$, if neutrons with kinetic energy not exceeding $120 \mathrm{MeV}$ interact at the top of the detector, the track of protons will be fully contained in the apparatus. However, if n-p scattering occurs in the lower part of the detector, the recoil protons will escape by crossing one anticounter plane. This means the anticounter is triggered and the neutron event will not be recorded, resulting in a geometrical factor dependent on energy. Furthermore, the nuclear interaction cross-section also depends on the energy of neutrons, which imposes a greater energy dependence on detection efficiency.

We actually obtained detection efficiency $(\varepsilon)$ using the Monte Carlo method and the Geant4 program, during which the collisions between neutrons and the carbon target were also taken into account. The detection efficiency of neutrons for vertical incidence without using any anti-coincidence panel can be approximated by $\varepsilon=3.45 \times E[\mathrm{MeV}]^{-0.7118}$. For example, neutrons with incident kinetic energy of $100 \mathrm{MeV}$ is expected to be detected by an efficiency of 0.13 or $13 \%$. 


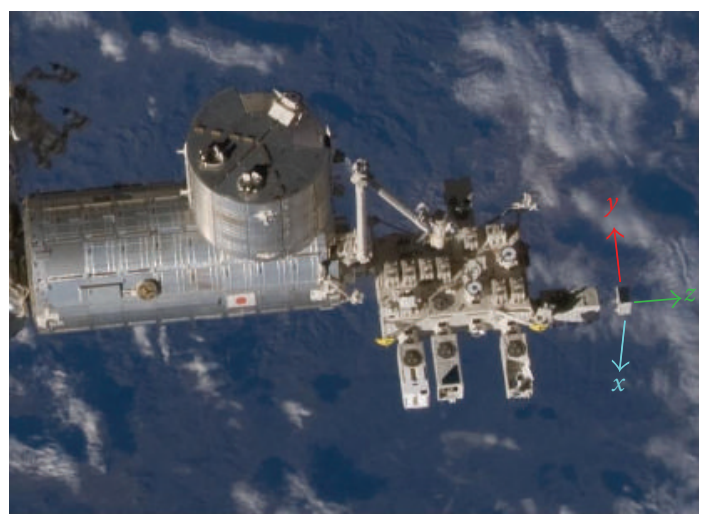

(a)

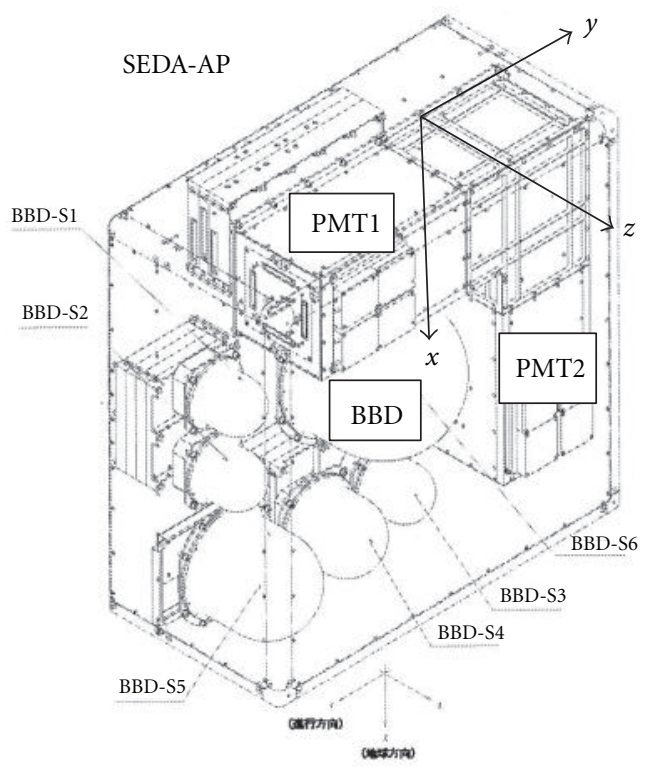

(b)

FIGURE 3: (a) The photograph of Japan Exposure module onboard the International Space Station. The coordinate of the FIB sensor is drawn on by the blue arrows ( $x$ coordinate blue, $y$ coordinate in red, and $z$ coordinate by green), (b) An FIB sensor was mounted on SEDA-AP together with BBD. Proton tracks are measured by a photomultiplier (PMT2) that looks the scintillation fibers from the bottom side ( $z-y$ plane), while for the $x-z$ plane the other photomultiplier (PMT1) is used looking from the proceeding section of the ISS.

For the incidence with $\theta=10,20$, and $30^{\circ}$, the coefficient 3.45 is replaced by $3.1,2.9$, and 2.6 , respectively. However, another condition has been applied to data analysis, whereby the minimum energy deposited in the sensor exceeds $35 \mathrm{MeV}$. According to the Monte Carlo calculation, the detection efficiency $(\varepsilon)$ can be expressed as $\varepsilon=1.15 \times(E-25[\mathrm{MeV}])$ $\times E[\mathrm{MeV}]^{-1.8}$. The results are given in Figure 4. In fact, the detection efficiency $(\varepsilon)$ could be approximately expressed by a constant value of 0.021 (almost 2\%) over a wide energy range of incident neutrons where $E_{\mathrm{n}}=50-120 \mathrm{MeV}$. We took account of these efficiencies when obtaining the energy spectrum of neutrons.

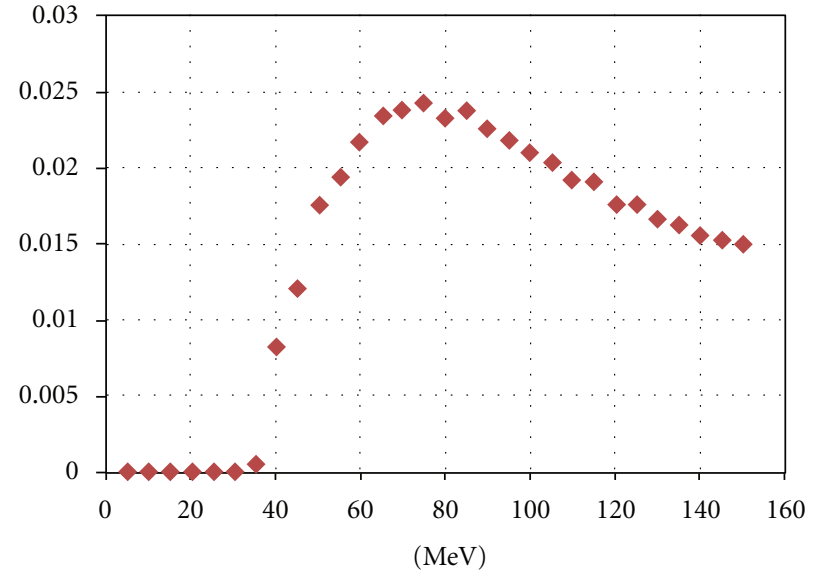

FIgURE 4: The detection efficiency of the FIB sensor for neutrons as a function of incident energy. The vertical value of 0.02 corresponds to the detection efficiency of $2 \%$. The curve was obtained by the Geant 4 program, taking account of the collision processes of neutrons with the proton or carbon target inside the scintillation fibers. The anticounter trigger condition was also taken into consideration.

\section{Measurement of Neutrons on the ISS}

This section presents the actual neutron measurement results obtained on the ISS. Figure 5 shows a typical event detected by the NEM sensor. The image on the right was taken by the PMT located in the proceeding section of the ISS ( $x-z$ plane), while that on the left is a photo taken from underneath the sensor ( $z-y$ plane) (i.e., looking up from Earth, see Figure 3(b)). The $z-y$ sensor points upward toward Earth and the $z$-direction points towards the opposite side of the pressurized ISS module. The color represents the amount of energy deposited in each scintillating bar of dimensions of $6 \times 3 \times 96 \mathrm{~mm}$. Figure 6 presents the counting rate of the FIB on March 7, 2011. Each panel of Figure 6 shows from the top to the bottom, the position of ISS, the strength of the magnetic field, the counting rate per minute and the integral counting rate, respectively. The satellite observed the solar flare after 20:02 UT.

As evidence that the FIB detector has been working stably, the energy spectrum of neutron-converted-protons is given in Figure 7 . The graph has been made by analyzing all data collected during January 1, 2010 and July 31, 2010. The observed proton spectrum can be expressed by a power law with a differential power index of -1.75 within the proton energy range where $E_{\mathrm{p}}=45-85 \mathrm{MeV}$. The data taken over the SAA region were excluded.

The trigger rate for neutrons was 0.22 counts $/ \mathrm{sec}$ on average (see Figure 6 the third panel) and 20 counts/sec over the South Atlantic Anomaly (SAA) region, which is about 90 times greater than anywhere else. The orbital average value 0.22 counts/sec is obtained after excluding the counting over the SAA. We have also measured neutron energy spectra using the range method. This may be the first time the energy of neutrons in space has been measured using the range method. As the trigger system occasionally encounters 


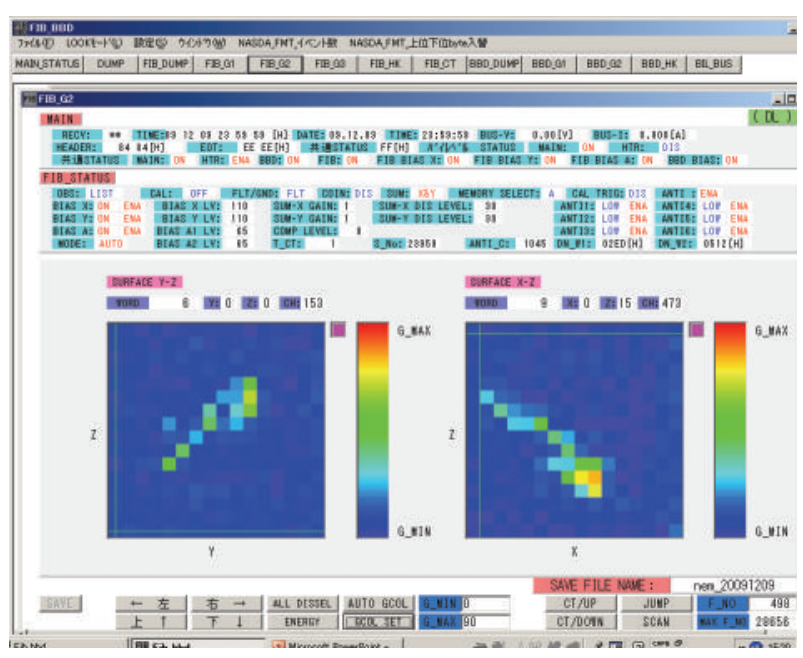

FIgURE 5: A typical background neutron event. The neutrons enter from the preceding section of the ISS (small to large in the horizontal scale $y$ of the left side picture) and from the top to the bottom (small to large of the right side picture $x$ in horizontal axis). The incident neutrons are converted into protons inside the scintillator. The energy deposit in each bar is color-coded. At the termination point of the track, the Bragg peak can be recognized. The G_min and G_max correspond to the ADC channel 0 and 90. The linear ADC has a range of 256 channels and the dip and peak correspond to 11 (dark blue) and 22 (light blue) respectively induced by the minimum ionizing particles. Orange spot corresponds to about channel 70 .

problems with memory saturation over the SAA region, it began recording only on-off data for each channel. At the end of this section, we mention the possibility of electron detection by this sensor. Using information on ionization loss $(-d E / d X)$, each of the electrons is separated from the neutron-converted-protons. The internal sensor can detect electrons with energy of between 2.5 and $30 \mathrm{MeV}$, as a thin track. Figure 8 shows a candidate electron track.

\section{Solar Neutrons Associated with M-Class Solar Flares}

4.1. Search for Solar Neutrons by the FIB Detector. According to the calculations of Imaida et al. [63] and Watanabe [64], the typical event rate induced by solar neutrons is expected to be within the range 10 to 1,000 counts/sec in FIB. As mentioned earlier, the background rate is as low as 0.22 counts/sec, making it possible to detect all solar neutrons exceeding this level. Between September 2009 and February 15, 2011, no solar flare occurred with intensities exceeding the X-class. However, during the period February 6 to 8 , 2010, four large M-class solar flares were observed, hence we analyzed the NEM data recorded at the time. Fortunately, during all four peaks of X-ray intensity, the satellite was flying over the daylight side of Earth. The peaks in X-ray intensity were observed by the GOES satellite on February 6 at 18:59 UT (M2.9), February 7 at 02:34 UT (M6.4), February 8 at $07: 53$ UT (M4.3), and again on February 8 at
13:47 UT (M2.0). Although we carefully searched the NEM records, there was no evidence of neutrons arriving from the Sun during any of these periods.

4.2. List of Flares and Search Conditions. We searched solar neutrons for every solar flare of GOES X-ray intensity exceeding $\mathrm{M} 2$, the results of which are summarized in Table 1. The first to third columns correspond to the event date, peak X-ray intensity, and flare class, respectively. The fourth column indicates the ISS location, namely whether on the night $(\mathrm{X})$ or day $(\mathrm{O})$ side of Earth. The fifth column indicates whether solar neutrons are involved in the data. The $\bigcirc \rightarrow \mathrm{X}$ and $\mathrm{X} \rightarrow \bigcirc$ notations in the fourth column indicate that the ISS was moving from the sunny side to the occultation side or vice versa 30 minutes from the peak flare time. A ? mark in the fifth column indicates a possible neutron signal with statistical significance less than $3 \sigma$.

Thanks to past observations, neutrons are known to be typically produced when the X-ray intensity peaks, and are observed within 30 minutes of this time via an X-ray detector $[3-5,31-33,45]$. Solar neutrons with energy of $35 \mathrm{MeV}$ need 23 minutes more than light to travel from the Sun to Earth. Of course, the maximum time observed by the GOES $\mathrm{X}$-ray detector does not always correspond to the neutron production time by the accelerated protons. Neutron energy of $35 \mathrm{MeV}$ corresponds to the minimum energy for tracking protons in the FIB detector. By taking these conditions into account, we set a data analysis time of 30 minutes and then searched for neutrons coming from the Sun.

4.3. Background. Before introducing the actual neutron events, let us briefly describe the background. As shown in the third panel of Figure 6, when the ISS approaches the northern or southern polar regions from the Equator, the neutron counting rate of the FIB detector increases from 0.04 to 0.5 counts/sec. The ISS completes an orbit around Earth every 90 minutes. Therefore, should solar neutrons arrive when the ISS passes over the Equator, high quality data are obtained. However, it also passes over either side of the polar regions during each 45-minute period.

For this period, the FIB detector demonstrates new functions to discriminate background and squeeze signals. We assume that the direction of protons induced by solar neutronswas observed within a cone with an opening angle of about 45 degrees relative to the direction of the Sun. By applying a simple acceptance calculation $\left(1 / 2^{*} \pi\right.$ steradian $/ 4 \pi$ steradian $=1 / 8$ ), the background may be reduced to $1 / 8$. We can therefore identify neutron signals under a background level of 0.028 counts/sec $(=0.22 / 8)$. In other words, when the intensity of solar neutrons is weaker than 0.028 counts/sec, solar neutrons will become far more difficult to detect.

4.4. Actual Event Observed on March 7, 2011. On March 7, 2011, in association with the M3.7 flare, a possible signal of solar neutrons was captured by the FIB detector and more than 54 proton events were identified as coming from the direction of the Sun. In Figure 9, we present a distribution of 
TABle 1: The list of solar flares with X-ray intensity exceeding $X>2.0$. The first to third columns of Table 1 correspond to the event date, maximum time of X-ray intensity, and flare size, respectively. The fourth column indicates the ISS location regarding whether on the shadow or sunny side of Earth (X or $\bigcirc$ ). The fifth column indicates whether solar neutrons are involved in the data. The $\bigcirc \rightarrow X$ and $X \rightarrow \bigcirc$ notations in the fourth column indicate that the ISS was moving from the sunny side to the shadow side or vice versa 30 minutes from the peak flare time. The ? mark of the fifth column indicates a possible neutron event with statistical significance of less than $3 \sigma$.

\begin{tabular}{|c|c|c|c|c|}
\hline Date & $\begin{array}{l}\text { Max. } \\
\text { X-ray time }\end{array}$ & Class & Satellite position & $\begin{array}{l}\text { Neutron } \\
\text { existence }\end{array}$ \\
\hline Feb. 6, 2010 & $18: 59$ & M2.9 & Sun side $\bigcirc$ & Neutron X \\
\hline Feb. 7, 2010 & $02: 34$ & M6.4 & Sun side $\bigcirc$ & Neutron X \\
\hline Feb. 8, 2010 & $07: 43$ & M4.0 & Sun side $\bigcirc$ & Neutron X \\
\hline Feb. 8, 2010 & $13: 47$ & M2.0 & Sun side $\bigcirc$ & Neutron X \\
\hline Feb. 12, 2010 & $13: 47$ & M2.0 & Sun side $\bigcirc$ & Neutron X \\
\hline Feb. 13, 2011 & $17: 38$ & M6.6 & Eclipse X & Neutron X \\
\hline Feb. 15, 2011 & $01: 44$ & $\mathrm{X} 2.2$ & Eclipse X & Neutron X \\
\hline Feb. 18, 2011 & $10: 11$ & M6.6 & Sun side $\bigcirc$ & Neutron? \\
\hline Feb. 24, 2011 & $07: 35$ & M3.5 & Eclipse X & Neutron X \\
\hline Mar. 7, 2011 & $20: 12$ & M3.7 & Sun side $\bigcirc$ & $\begin{array}{c}\text { Neutron } \\
\bigcirc\end{array}$ \\
\hline Mar. 8, 2011 & $10: 44$ & M5.3 & Eclipse X & Neutron X \\
\hline Mar. 8, 2011 & $18: 28$ & M4.4 & Eclipse X & Neutron X \\
\hline Mar. 9, 2011 & $23: 23$ & $\mathrm{X} 1.5$ & Eclipse X & Neutron X \\
\hline Jun. 7, 2011 & 06:30 & M2.5 & $\bigcirc \rightarrow \mathbf{X}$ & $\begin{array}{c}\text { Neutron } \\
\bigcirc\end{array}$ \\
\hline Jul. 30, 2011 & 02:09 & M9.3 & Sun side $\bigcirc$ & Neutron? \\
\hline Aug. 3, 2011 & $13: 48$ & M6.0 & Sun side $\bigcirc$ & Neutron X \\
\hline Aug. 4, 2011 & $03: 57$ & M9.3 & Sun side $\bigcirc$ & $\begin{array}{c}\text { Neutron X } \\
?\end{array}$ \\
\hline Aug. 8, 2011 & $18: 10$ & M3.5 & Sun side $\bigcirc$ & Neutron? \\
\hline Aug. 9, 2011 & $03: 54$ & M2.5 & Sun side $\bigcirc$ & Neutron? \\
\hline Aug. 9, 2011 & 08:05 & X6.9 & Sun side $\bigcirc$ & Neutron X \\
\hline Sep. 6, 2011 & $01: 50$ & M5.3 & Sun side $\bigcirc$ & Neutron X \\
\hline Sep. 6, 2011 & $22: 20$ & $\mathrm{X} 2.1$ & Sun side $\bigcirc$ & Neutron X \\
\hline Sep. 7, 2011 & $22: 38$ & $\mathrm{X} 1.8$ & Sun side $\bigcirc$ & $\begin{array}{c}\text { Neutron } \\
\text { X? }\end{array}$ \\
\hline Sep. 8, 2011 & $15: 46$ & M6.7 & Sun side $\bigcirc$ & $\begin{array}{c}\text { Neutron } \\
\bigcirc\end{array}$ \\
\hline Sep. 9, 2011 & $06: 11$ & M2.7 & $\bigcirc \rightarrow X$ & Neutron X \\
\hline Sep. 22, 2011 & $11: 01$ & $\mathrm{X} 1.4$ & $\bigcirc \rightarrow X$ & Neutron? \\
\hline Sep. 24, 2011 & 09:40 & X1.9 & $\mathrm{X} \rightarrow \bigcirc$ & Neutron X \\
\hline Sep. 24, 2011 & $13: 20$ & M7.1 & Sun side $\bigcirc$ & Neutron X \\
\hline Sep. 24, 2011 & 19:18 & M3.0 & Sun side $\bigcirc$ & $\begin{array}{c}\text { Neutron } \\
\bigcirc\end{array}$ \\
\hline Sep. 24, 2011 & $20: 36$ & M5.8 & Sun side $\bigcirc$ & Neutron X \\
\hline Sep. 25, 2011 & $02: 33$ & M4.4 & Eclipse $(X \rightarrow \bigcirc)$ & Neutron? \\
\hline Sep. 25, 2011 & $04: 50$ & M7.4 & Eclipse $(X \rightarrow \bigcirc)$ & Neutron? \\
\hline Sep. 25, 2011 & $15: 33$ & M3.7 & Sun side $\bigcirc$ & Neutron X \\
\hline Sep. 26, 2011 & 05:08 & M4.0 & Sun side $\bigcirc$ & Neutron X \\
\hline Sep. 26, 2011 & $14: 45$ & M2.6 & Sun side $\bigcirc$ & Neutron X \\
\hline Oct. 2, 2011 & 00:50 & M3.9 & Sun side $\bigcirc$ & Neutron X \\
\hline
\end{tabular}


Table 1: Continued.

\begin{tabular}{|c|c|c|c|c|}
\hline Date & $\begin{array}{c}\text { Max. } \\
\text { X-ray time }\end{array}$ & Class & Satellite position & $\begin{array}{l}\text { Neutron } \\
\text { existence }\end{array}$ \\
\hline Nov. 2, 2011 & $22: 01$ & M4.3 & Sun side $\bigcirc$ & Neutron X \\
\hline Nov. 3, 2011 & $20: 27$ & X1.9 & Sun side $\bigcirc$ & $\begin{array}{c}\text { Neutron } \\
\bigcirc\end{array}$ \\
\hline Nov. 5, 2011 & 03:35 & M3.7 & Sun side $\bigcirc$ & Neutron X \\
\hline Dec. 25, 2011 & $18: 16$ & M4.0 & Eclipse X & Neutron X \\
\hline Jan. 19, 2012 & $16: 05$ & M3.2 & Sun side $\mathrm{X} \rightarrow \bigcirc$ & Neutron X \\
\hline Jan. 23, 2012 & 03:59 & M8.7 & $\begin{array}{c}\text { Sun side } \\
\bigcirc \rightarrow \mathrm{X}\end{array}$ & $\begin{array}{c}\text { Neutron } \\
\bigcirc\end{array}$ \\
\hline Jan. 27, 2012 & $18: 37$ & $\mathrm{X} 1.7$ & Sun side $\bigcirc$ & Neutron X \\
\hline Mar. 2, 2012 & $17: 46$ & M3.3 & Sun side $\bigcirc$ & Neutron? \\
\hline Mar. 4, 2012 & $10: 45$ & M2.0 & Eclipse X & Neutron X \\
\hline Mar. 5, 2012 & 04:05 & $\mathrm{X} 1.1$ & $\mathrm{X} \rightarrow \bigcirc$ & Neutron? \\
\hline Mar. 7, 2012 & $00: 24$ & $\mathrm{X} 5.4$ & $\bigcirc \rightarrow X$ & Neutron X \\
\hline Mar. 9, 2012 & 03:45 & M6.3 & Sun side $\bigcirc$ & Neutron? \\
\hline Mar. 10, 2012 & $17: 50$ & M8.4 & Eclipse X & Neutron X \\
\hline Mar. 13, 2012 & $17: 25$ & M7.8 & Sun side $\bigcirc$ & Neutron? \\
\hline May. 9, 2012 & $12: 32$ & M4.7 & Sun side $\bigcirc$ & Neutron X \\
\hline May. 9, 2012 & $21: 05$ & M4.1 & Sun side $\bigcirc$ & Neutron X \\
\hline May. 10, 2012 & 04:18 & M5.7 & Sun side $\bigcirc$ & Neutron X \\
\hline May. 17, 2012 & $01: 47$ & M5.1 & Sun side $\bigcirc$ & Neutron X \\
\hline Jul. 2, 2012 & $10: 50$ & M5.6 & Eclipse X & Neutron X \\
\hline Jul. 2, 2012 & 20:05 & M3.8 & Eclipse X & Neutron X \\
\hline Jul. 4, 2012 & 09:55 & M5.3 & Sun side $\bigcirc$ & Neutron X \\
\hline Jul. 4, 2012 & $22: 05$ & M4.6 & Sun side $\bigcirc$ & Neutron X \\
\hline Jul. 5, 2012 & $11: 40$ & M6.1 & Sun side $\bigcirc$ & Neutron X \\
\hline Jul. 6, 2012 & $23: 05$ & $\mathrm{X} 1.1$ & $\mathrm{X} \rightarrow \bigcirc$ & Neutron X \\
\hline Jul. 8, 2012 & $16: 30$ & M6.9 & Eclipse X & Neutron X \\
\hline Jul. 17, 2012 & $17: 15$ & M1.7 & Sun side $\bigcirc$ & Neutron X \\
\hline Jul. 19, 2012 & 05:58 & M7.7 & Sun side $\bigcirc$ & Neutron X \\
\hline
\end{tabular}

the arrival direction of those 54 events over the background at the same time.

The statistical significance of the event was $6.8 \sigma$ (based on the Li-Ma method). We regard those protons as being produced by solar neutrons inside the FIB sensor. Figure 10 shows an example of actual event involving "solar" neutrons. The direction of the Sun was to the lower-left side for pictures on the left $(y-z$ coordinate) in Figure 10, while the solar direction was to the lower-center side for pictures on the right ( $x-z$ coordinate). The track in Figure 10 corresponds to proton energy of $44 \mathrm{MeV}$. The event was observed at 20:10:26 UT and departed time from the Sun is estimated as to be $19: 50: 38 \mathrm{UT}$.

Figure 11 presents the arrival time distribution of neutron-induced protons. The horizontal axis represents minutes after 20:00 UT. The red curve represents the result, with equivalent analytical conditions applied to the data obtained exactly 90 minutes later. As can be seen, contamination from the background to the possible signal involved time is relatively small. Figure 11 also involves important information. The final candidate of neutrons arrived near
Earth 11 minutes later than the first one. The energy of neutrons was $44 \mathrm{MeV}$, so we can estimate the latest departure time from the Sun to be after 19:52:36 UT.

The FIB sensor has a function of measuring the energy of neutron-induced protons. Therefore the flight time of neutrons can be estimated. The result is shown in Figure 12. In making Figure 12, the events are used that were emitted in the forward cone with an opening angle of less than 20 degrees. Figure 12 suggests that neutrons were emitted from the Sun during 19:41 and 19:54 UT. It is worthwhile to note that the highest channel $(7-20 \mathrm{MeV})$ of the RESSHI satellite observed an enhancement during 19:42-20:05 UT. The time of peak intensity of the hard X-rays observed by the RHESSI satellite (50-100 keV) for this flare was 20:02 UT.

4.5. An Interpretation on the Time of Flight Distribution of Neutrons. The neutron-induced protons of Figure 12 do not necessarily reflect correct flight time of neutrons from the Sun. There is a possibility that the first bump around 19: 48 UT was induced by the n-C scattering. Therefore we 
FIB count profile

(2011/03/07-19:30:00-2011/03/07-20:20:00)
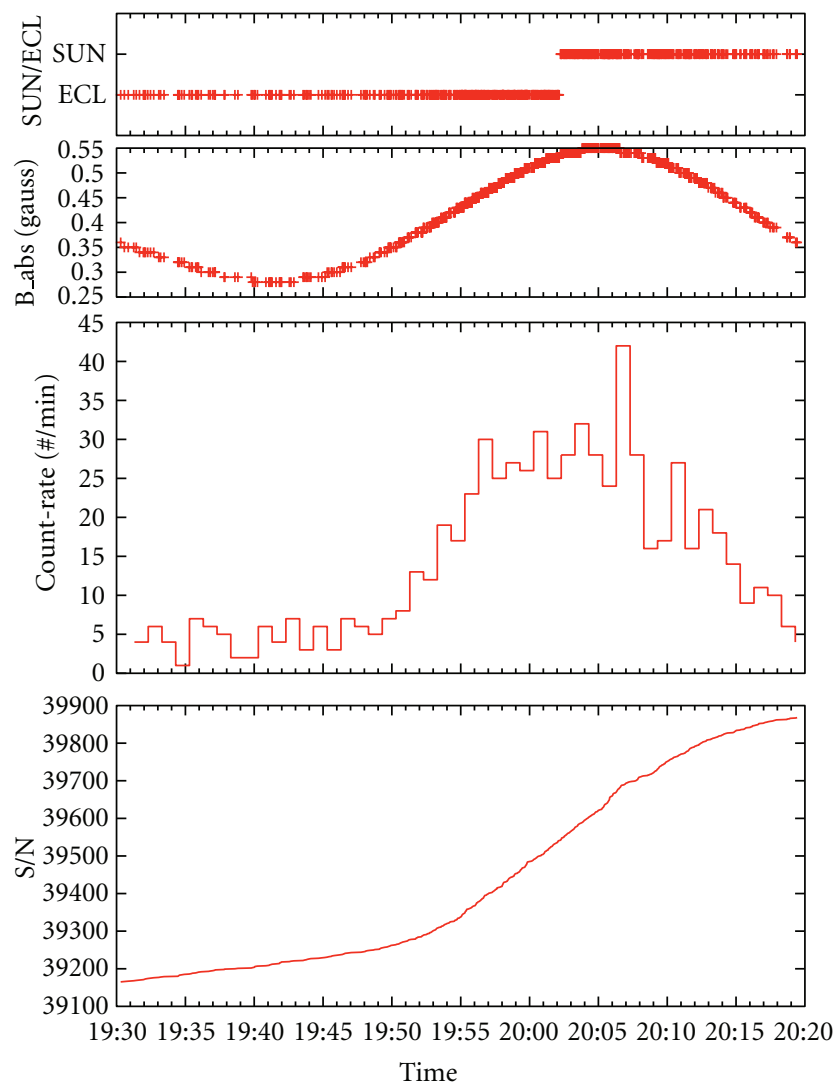

FIGURE 6: From the top to the bottom, each figure corresponds to (1) the location of the satellite, whether it was over the day side of the Earth or the night side, (2) the strength of the magnetic field measured by SEDA-AP, (3) the differential event rate per minute, and (4) the integral counting rate with Universal Time. The SUN or ECL of the top panel represents whether the satellite was on the day side (1) or the night side (0) of the Earth, respectively. The satellite observed the Sun after 20:02 UT. Every 45 minute, the satellite passes over the Equator and approaches both Polar Regions. The FIB is an omnidirectional sensor and detects neutrons from all directions. The peak during 20:06-20:07 UT may be induced by solar neutrons.

present in the $E_{\mathrm{p}} / E_{\mathrm{n}}$ distribution Figure 13. To make this figure an assumption is made that those neutrons were produced at the same time on the solar surface at 19:52 UT. We could then evaluate their energy $E_{\mathrm{n}}$ from the flight time to the top of the atmosphere. $E_{\mathrm{p}}$ can be obtained for each neutron-induced proton using the range method by the FIB sensor itself. Interestingly, about more than two-thirds of incoming neutron energy is converted into protons, which suggests that some of the break-up neutrons of the carbon target induced by neutrons may have escaped from the sensor to outside. This assumption has been supported by our Monte Carlo calculation based on the Geant- 4 program. According to the MC calculation, the mean proton energy $\left\langle E_{\mathrm{p}}\right\rangle$ observed by the FIB sensor can be written by the inverse square root of the incident energy of neutrons $E_{\mathrm{n}} ;\left\langle E_{\mathrm{p}}\right\rangle$

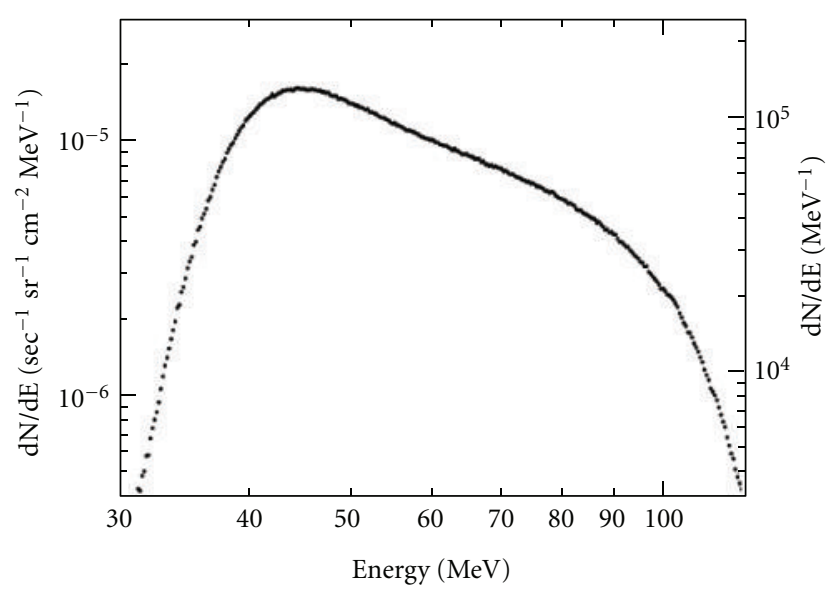

FIGURE 7: Energy spectrum of protons recorded in the FIB sensor during January 1-July 31, 2010. The data obtained over the SAA region is unrelated to this data. The left side axis represents the differential flux of neutron-induced protons denoted by the unit of $\left(/ \mathrm{sec} \cdot \mathrm{sr} \cdot \mathrm{cm}^{2} \cdot \mathrm{MeV}\right)$.

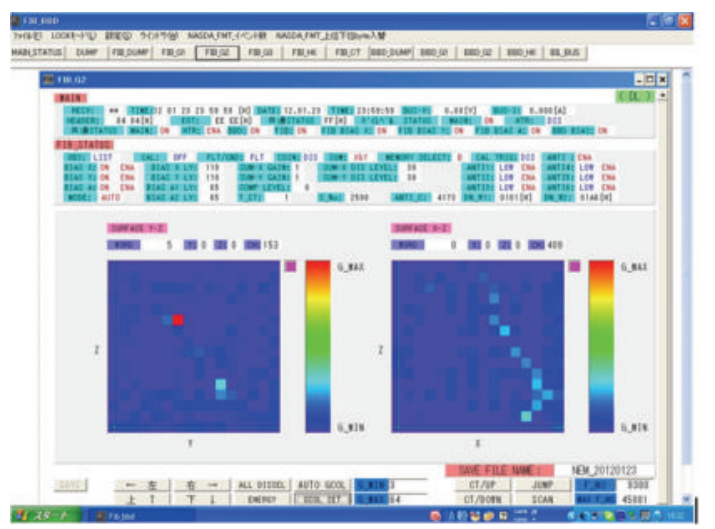

Figure 8: A candidate electron track. A photon enters from the top of the sensor ( $y-z$ plane). An electron apparently deposited energy inside one vertical bar (where the red spot denotes a large deposit of energy) while running vertically, thus enabling the recording of a long thin track in the $x-z$ plane. Since the ionization loss of electrons is smaller than that of protons, thin tracks are expected.

$=0.55 * E_{\mathrm{n}} / \operatorname{Sqrt}\left(E_{\mathrm{n}} / 100 \mathrm{MeV}\right)$. For example neutrons with incident energy of $100 \mathrm{MeV}$ will be observed as protons with an average energy of $55 \mathrm{MeV}$ in the FIB sensor. However we must note here since the event number of the dip between 19:49-19:50 UT is not enough, so this dip has not been confirmed statistically.

Taking account of these facts (1) the angular distribution presented in Figure 9, (2) the production time distribution of neutrons (presented in Figure 12) coincides with the duration of the flare start time (19:43 UT) and the peak time $(20: 12 \mathrm{UT})$ (measured by the GOES X-ray detector), (3) the coincidence of the flight time of the last event with an assumption that they were produced around 19:52 UT, and (4) no such enhancement was observed in the data after 90 minutes later, it would be the most natural to think this 


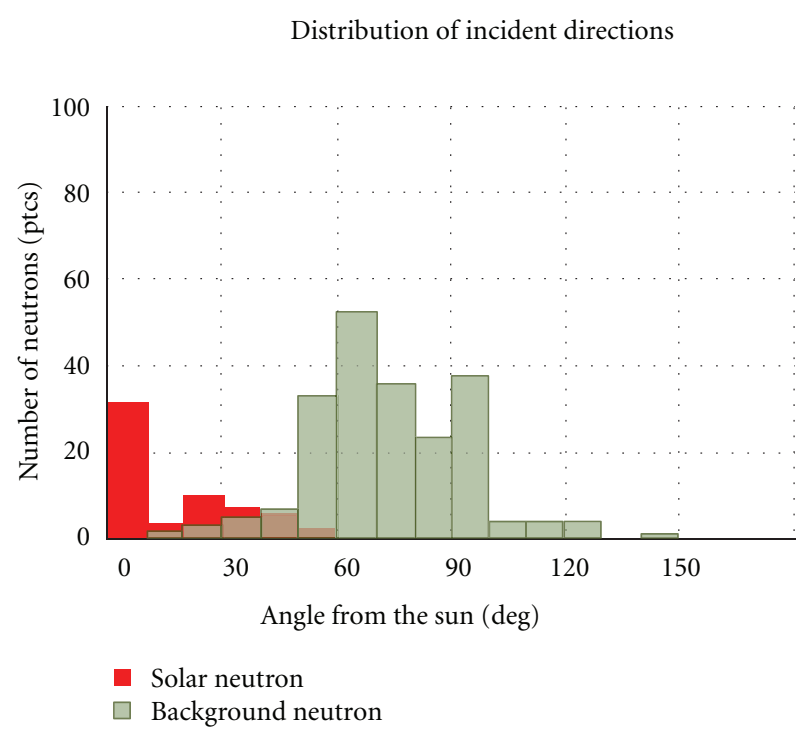

(a)

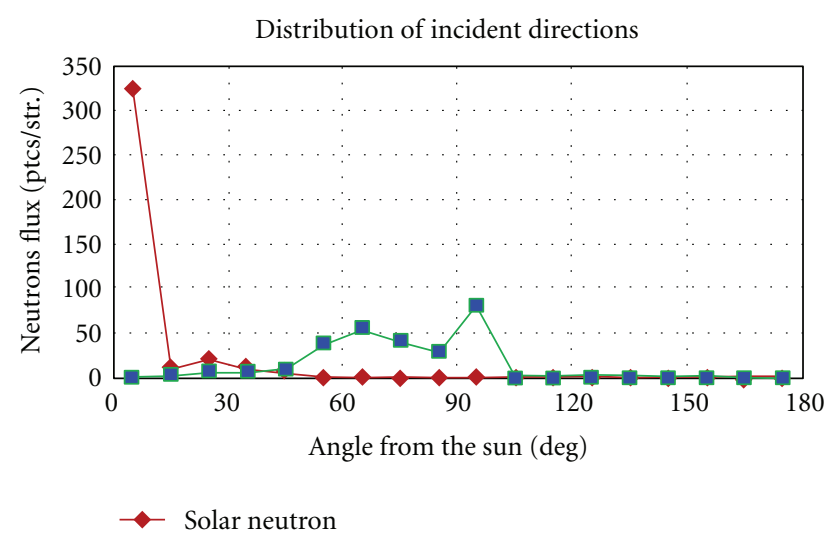

(b)

FIgURE 9: (a) The arrival directions of neutrons, as observed by the FIB sensor during the period 19:59:43-20:16:34 UT. Neutrons coming from the Sun (red) are shown separately from the background neutrons (dark green), but those neutrons are actually observed together by the FIB sensor. The number of events recorded during the above period was 364 , and for 44 , it was difficult to determine the arrival direction due to low energy. A clear peak of neutrons can be identified within the region 0-20 degrees from the solar direction. Meanwhile, the candidates between 20 and 40 degrees may be neutrons from the Sun, induced by $\mathrm{n}-\mathrm{C}$ scattering and also distributed at a relatively wide angle due to the limited angular resolution of the FIB sensor for the lowest energy neutrons (about $35 \mathrm{MeV}$ ). The bump of the background within the region 40-100 degrees from the solar direction corresponds to the background neutrons produced in the material of JEM by galactic cosmic rays. (b) The same data as used in the plot of (a). However the data were normalized by the solid angle. A sharp peak can be recognized for neutrons from the solar direction (red), while the background neutrons distribute in different angles presented by the green curve. The vertical value represents $d N / d \Omega$. Total number of events during 20:02-20:16 UT was 273 and 54 for the background and for the signal respectively.

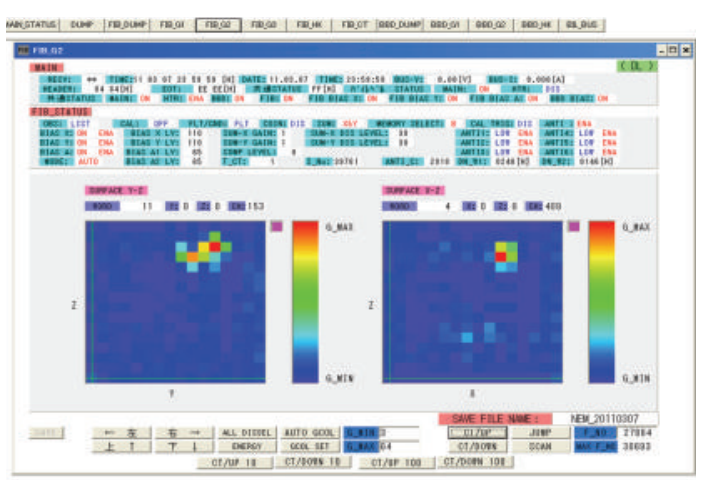

FIGURE 10: Solar neutron the event number 39,761 detected by the FIB sensor at $20: 10: 26$ UT on March 11,2011. The solar direction was located toward the lower left side in $y$ - $z$ plane (the left side panel), while in the right side panel the solar direction was down ward almost vertically. The energy of the track is estimated as $(44 \pm 5) \mathrm{MeV}$ and the neutron may be emitted around 19:50:38 UT at the Sun, if the track was induced by the $\mathrm{n}-\mathrm{p}$ collision.

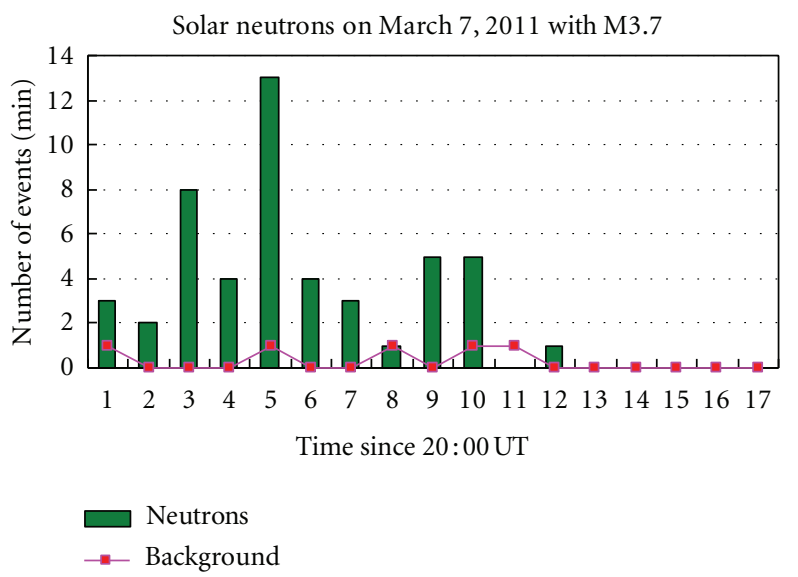

FIgURE 11: The arrival time distribution of neutron-induced protons. The horizontal axis represents minutes after 20:00 UT. The green histogram corresponds to neutrons from the solar direction. The red curve represents the result, with equivalent analytical conditions applied to the data obtained exactly 90 minutes later.

event as following: those neutrons were produced at the Sun in association with the M3.7 solar flare.

4.6. Other Events. Three months later, the FIB detector observed another neutron event. This time the flare's intensity was only M2.5, hence far below the X-class scale. A total of 36 neutron events were identified. The statistical significance of the event was $5.8 \sigma$. The enhancement was observed during 06:21 and 06:41 UT. Figure 14 shows the angular distribution of those events from the solar direction. On this event, the direction of the Sun was the opposite side of the pressurized module of ISS. So the identification from the background was easily made. The discrimination was also made by confirming the Bragg peak.

We observed three more candidate solar neutron events up to the end of July 2012: the flare events on Sep. 24, 2011 


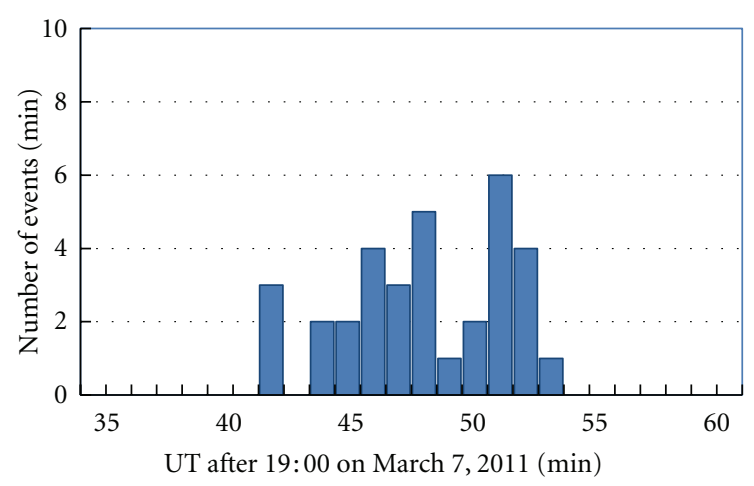

Figure 12: The departure time distribution of solar neutrons. The histogram has been made being based on neutrons detected within a cone angle of 20 degrees from the solar direction. From the energy, the time of flight was calculated and converted the value into the departure time of neutrons from the Sun. There were two bumps around 19:48 UT and 19:52 UT.

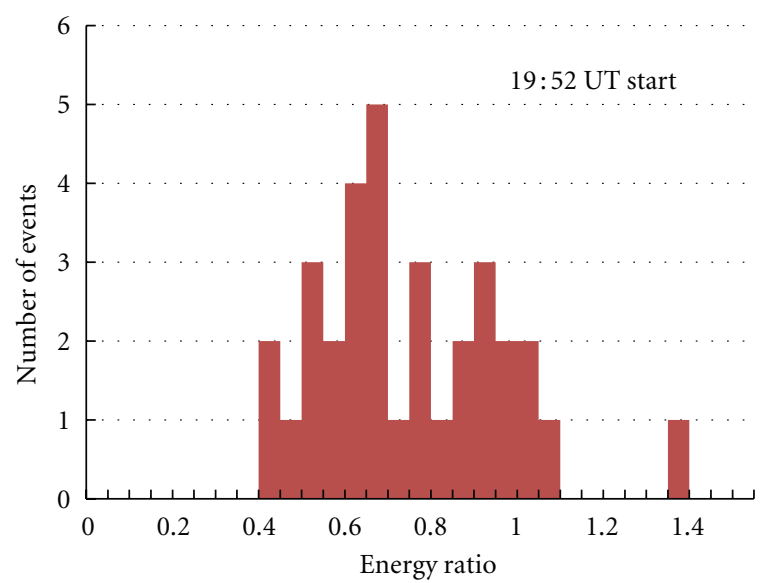

Figure 13: Energy ratio between converted protons $\left(E_{\mathrm{p}}\right)$ versus incident neutrons $\left(E_{\mathrm{n}}\right)$. Proton energy $\left(E_{\mathrm{p}}\right)$ was measured using the range method by the FIB sensor, while neutron energy was estimated by flight time. We assumed that the solar neutrons departed at the same time (19:52 UT) from the Sun. There is an event beyond 1.0. However taking account of the energy resolution of the sensor, it sometimes happens.

(M3.0), Nov. 30, 2011 (X1.9), and Jan. 23, 2012 (M8.7). As few as 27 events were observed on September 24, compared to the 42 events on Nov. 30, 2011, while a total of 50 neutrons were observed in association with the flare event on Jan. 23, 2012. Those results are under preparation and will be published in elsewhere. Table 2 summarizes those observation results, along with other observations.

\section{Discussions and Comparisons with Other Observations}

A solar neutron event was observed on March 7, 2011, followed by another on June 7, 2011. Both provided a new perspective regarding the production process of solar neutrons. To date, neutrons have been observed on the occasion
TABLe 2: A score table between each satellite. The numbers of solar neutron events, from first to third, represent protons induced by neutrons, the incidents of which are consistent with those coming from the Sun, low-energy events difficult to identify as solar neutrons and ambiguous events due to low energy respectively. The marks $\bigcirc, \Delta$, and $\times$ imply that each satellite was passing over the day side $(\bigcirc)$, partial day side $(\Delta)$ or night side $(\times)$ of Earth, respectively. The event observed on September 8, 2011, was not involved in this table, because it was observed near the SAA.

\begin{tabular}{llcccc}
\hline \multicolumn{2}{c}{ ISS SEDA-FIB neutron event } & Hinode & RHESSI & Fermi-LAT \\
\hline Mar. 7, 2011 & M3.7 & $54,35,28$ & $\times$ & $\bigcirc$ & $\Delta$ \\
Jun. 7, 2011 & M2.5 & $86,2,20$ & $\bigcirc$ & $\bigcirc$ & $\bigcirc$ \\
Sep. 24, 2011 & M3.0 & $27,2,20$ & $\bigcirc$ & $\bigcirc$ & $\Delta$ \\
Nov. 3, 2011 & X1.9 & $42,15,8$ & $\Delta$ & $\times$ & $\times$ \\
Jan. 23, 2012 & M8.7 & $50,14,20$ & $\bigcirc$ & $\times$ & $\times$ \\
\hline
\end{tabular}

of strong solar flares with X-ray intensity of $\gtrsim X 10$ [65]. However, the present results indicate that even solar neutrons are produced by $\mathrm{M}$-class solar flares. The difference in X-ray intensity between X10 and M2.5 is about 40 times greater. The present results thus suggest that solar neutrons including M-class solar flares require a careful and complete search.

The SONG detector on board the Russian satellite CORONUS-F actually observed solar neutrons on three occasions in the solar cycle 23. However, the solar neutrons were only detected for X-class solar flares on August 25, 2001, October 28, 2003 and November 4, 2003 [66] respectively. Moreover, neutron monitors or solar neutron telescopes observed no solar neutrons from August 2009 to the end of July 2012, which may suggest that the soft X-ray flux measured by the GOES satellite does not necessarily correspond to the intensity of solar neutrons from the Sun. Although the link between this fact and the magnetic field structure [67] remains unclear, it remains a fascinating subject to be studied.

Another surprising fact is for such medium-class solar flares as those that occurred on March 7 (M3.7) and June 7, 2011 (M2.5), the LAT detector on board the Fermi satellite had observed long-lasting gamma-ray emissions. The emission of gamma rays with energies of $100 \mathrm{MeV}$ to $1 \mathrm{GeV}$ continued for more than 14 hours [47]. This marked a new discovery as the EGRET detector on board the CGRO satellite observed such long-lasting gamma-ray emissions with energy exceeding $150 \mathrm{MeV}$ for the flare event on June 11, 1991 [29]. The RHESSI satellite observed the flare event on March 7 from its start time to the peak time, although the time profile of hard X-rays showed no unusual features and had a normal shape. The telescope of the Solar Dynamical Observatory (SDO) observed a very interesting feature; Coronal Mass Ejection (CME) started before the flare observed by the GOES/RHESSI satellites. A prominent injection of hot plasma into the base of the CME via loops was also observed by the SDO telescope. The signal of neutrons detected by the SEDA-FIB might be produced after that at the top of the inverse U-shaped loop around 19:58 UT [37], while the long-lasting high-energy gamma rays of FERMI-LAT may be produced by the precipitation 
TABLE 3: Acomparison of solar neutron events observed by the SEDA-FIB detector with SMM event. The average trigger rate is simply obtained by dividing the total number of events by the observation time, while the mean flux is obtained taking the detection efficiency of both detectors into account ( 0.021 and 0.3 for SEDA and SMM) and also the detector area (100 and $450 \mathrm{~cm}^{2}$ for SEDA and SMM).

\begin{tabular}{lccccc}
\hline \multicolumn{2}{c}{ Date of events } & & Total number of events & Average trigger rate $(\mathrm{Hz})$ & $\mathrm{Mean}$ flux $\left(\mathrm{events} / \mathrm{cm}^{2} \mathrm{sec}\right)$ \\
\hline Mar. 7, 2011 & M3.7 & SEDA & 89 & 0.09 & 0.042 \\
Jun. 7, 2011 & M2.5 & SEDA & 88 & 0.06 & 0.029 \\
Jun. 21, 1980 & X2.5 & SMM & 2,000 & 2.0 & 0.13 \\
\hline
\end{tabular}

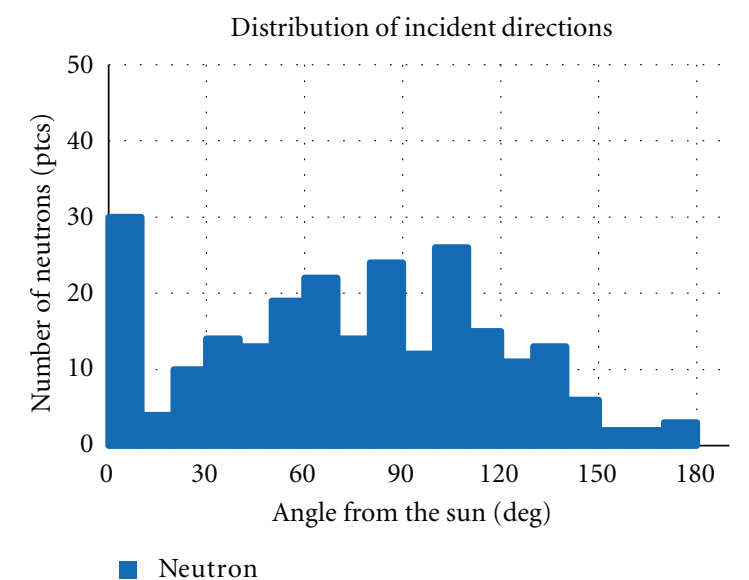

(a)

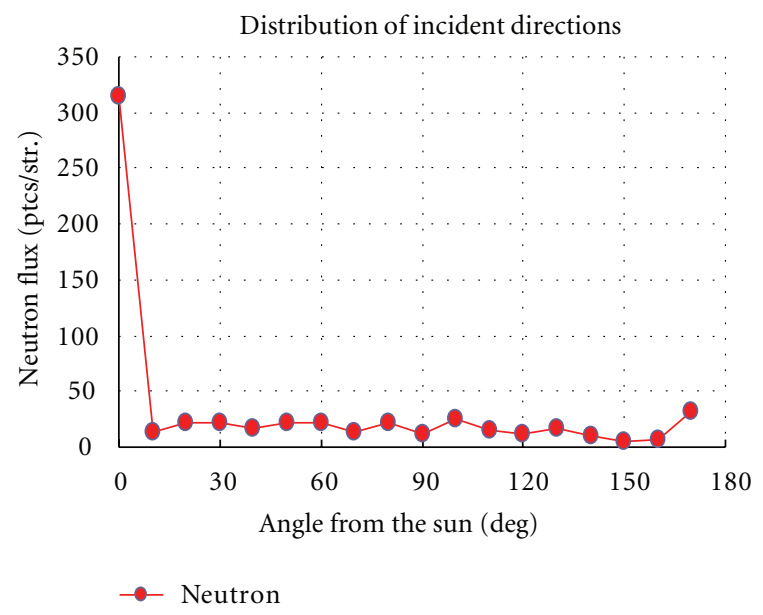

(b)

FIgURE 14: (a) The angular distribution of neutrons detected by the FIB sensor during 06:20 UT and 06:41 UT on June 7, 2011. Total number of neutrons detected above duration was 240. (b) The histogram of (a) is divided by the solid angle. The vertcical axis represents $d N / d \Omega$. A sharp peak is recognized at the solar direction, while beyond 10 degrees, almost flat distribution is seen.

of the accelerated protons on the solar surface. The activity continued for more than 10 hours [47].

For the solar flare event on June 7, 2011, hard X-ray data are available from the Fermi-GBM and RHESSI detectors. Once again, the telescope of the Solar Dynamical Observatory took a very interesting picture of this flare. The UV detector (sensitive to $171 \mathrm{~nm}$ ) observed the very large-scale precipitation of plasma onto the solar surface
$[53,54]$. In coincidence with this precipitation, protons trapped inside the upper "plasma bag" may precipitate over the solar surface. What resembles a "blow brush" over the reconnection point emits the high-energy protons confined in the plasma bag onto the solar surface, at which time the long-lasting gamma rays may be produced. However, the neutrons observed by the FIB detector may be produced when the protons accelerate, when magnetic loops are reconnected on top of the solar surface rather impulsively.

The UV telescope of SDO, however, did not observe any precipitation of plasma for the flare event on March 7, 2011, but instead a clear picture of the beginning of Coronal Mass Ejection (CME). After the seed of CME had been ejected, all loops under the CME started shining brightly. The protons were probably accelerated and neutrons possibly produced in one such loop via loop-to-loop interactions [68] when those protons struck the solar surface.

\section{Summary}

A new solar neutron detector was launched on July 16, 2009, on board the Space Shuttle Endeavour, and began operation at the ISS on August 25, 2009. The sensor can determine both the energy and arrival direction of neutrons. We measured some signals of solar neutron events in association with $\mathrm{M}$ class solar flares that occurred on March 7 and June 7, 2011. It is interesting to note that even for $\mathrm{M}$-class solar flares, we had better search solar neutrons by using both the satellite and ground level detectors.

The precipitation of plasma bubbles, the long-lasting emission of gamma rays, and the starting of CME seeds were among the interesting features observed together with these flares. The new data obtained certainly provides us with a new perspective on the process behind the production of gamma rays and neutrons at the Sun.

The FIB detector on board the ISS observed three further solar neutron events on Sep. 24, 2011 (M3.0), Nov. 30, 2011 (X1.9), and Jan. 23, 2012 (M8.7). More in-depth analysis is now being conducted in connection with other data. In Table 3, we compared the March 7 and June 7, 2011 events with the solar neutron event on June 21, 1980.

\section{Acknowledgments}

The authors acknowledge the crew of the Space Shuttle Endeavour who successfully mounted the SEDA-FIB detector on the ISS Kibo exposed facility. They also extend thanks to the members of the Tsukuba operation center of Kibo for taking the SEDA-FIB data every day. The authors also 
acknowledge Dr. Satoshi Masuda of Solar-Terrestrial Environment laboratory of Nagoya University and Professor Masahiro Hoshino of the University of Tokyo for valuable discussions on the solar flares of March 7 and June 7, 2011 and the acceleration mechanism of protons over the solar surface, respectively.

\section{References}

[1] S. E. Forbush, "Three unusual cosmic-ray increases possibly due to charged particles from the sun," Physical Review, vol. 70, no. 9-10, pp. 771-772, 1946.

[2] L. Biermann, O. Haxel, and A. Schlüter, "Neutral cosmic rays produced in the Sun," Zeitschrift für Naturforschung, vol. 6, no. 1, pp. 47-48, 1951.

[3] E. L. Chupp, D. J. Forrest, J. M. Ryan et al., "A direct observation of solar neutrons following The 0118 UT flare on 1980 June 21," The Astrophysical Journal, vol. 263, pp. L95L98, 1982.

[4] D. J. Forrest, E. L. Chupp, and J. M. Ryan, "Model calculations on fast solar neutrons and the $2.2 \mathrm{MeV}$ line emission from solar flares," in Proceedings of the 17th International Cosmic Ray Conference (ICRC '81), vol. 10, p. 5, 1981.

[5] E. L. Chupp, H. Debrunner, E. Flückiger et al., "Solar neutron emissivity during the large flare on 1982 June 3," The Astrophysical Journal, vol. 318, pp. 913-925, 1987.

[6] H. Debrunner, E. O. Flückiger, J. A. Loclwood, and D. J. Forrest, "The solar cosmic ray neutron event on June 3, 1982," in Proceedings of the 18th International Cosmic Ray Conference (ICRC '83), vol. 4, pp. 75-78, Bangalore, India, 1983.

[7] Y. E. Efimov, G. E. Kocharov, and K. Kudela, "On the solar neutrons observation on high mountain neutron monitor," in Proceedings of the 18th International Cosmic Ray Conference (ICRC '83), vol. 10, pp. 276-278, Bangalore, India, 1983.

[8] S. Shibata, K. Murakami, Y. Muraki et al., in Proceedings of the 22nd International Cosmic Ray Conference (ICRC '91), vol. 3, pp. 788-791, Dublin, Ireland, 1991.

[9] R. Bütikofer, E. O. Flückiger, L. Desorgher et al., "SONTELmeasurements at Gornergrat and environmental radioactivity," in Proceedings of the 28th International Cosmic Ray Conference (ICRC '03), vol. 7, pp. 4189-4192, Tsukuba, Japan, 2003.

[10] J. A. Simpson, "The cosmic ray nucleonic component: the invention and scientific uses of the neutron monitor," Space Science Reviews, vol. 93, no. 1-2, pp. 11-32, 2000.

[11] K. R. Pyle and J. A. Simpson, in Proceedings of the 22nd International Cosmic Ray Conference (ICRC 'O0), vol. 3, pp. 5356, Dublin, Ireland, 2000.

[12] M. A. Shea and D. F. Smart, "Fifty years of cosmic radiation data," Space Science Reviews, vol. 93, pp. 229-262, 2000.

[13] J. M. Clem and L. I. Dorman, "Neutron monitor response functions," Space Science Reviews, vol. 93, no. 1-2, pp. 335$359,2000$.

[14] R. Koga, G. M. Frye Jr., A. Owens, B. V. Deneby, O. Mace, and T. Jenkins, "A search for solar neutrons on a long duration balloon flight," in Proceedings of the 19th International Cosmic Ray Conference (ICRC '85), vol. 4, pp. 142-145, La Jolla, Calif, USA, 1985.

[15] R. S. Miller, J. R. Macri, M. L. McConnell, J. M. Ryan, E. Flueckiger, and L. Desorgher, "Nuclear instruments and methods in physics research section A: accelerators, spectrometers, detectors and associated equipment," in Proceedings of the 10th
Symposium on Radiation Measurements and Applications, vol. 505, pp. 36-40, 2003.

[16] J. Shimizu, in Proceedings of the 19th Workshop on Space Station Utilization, pp. 8.32-8.55, 1997.

[17] R. Chikaoka, "Minutes of the 12th Space development, Committee of the Ministry of Education, Science and Culture," Reports 13-1 and 12-3, 1997.

[18] M. McConnell, K. Bennett, D. Forrest et al., "Comptel observations of solar flare gamma-rays," Advances in Space Research, vol. 13, no. 9, pp. 245-248, 1993.

[19] M. McConnell, "High-energy solar phenomena-a new era of spacecraft measurements," in Proceedings of the AIP Conference, vol. 294, pp. 21-25, American Institute of Physics, New York, NY, USA, 1993.

[20] The Yohkoh HXT/SXT Flare Catalogue, Edited by J. Sato, M. Sawa, K. Yoshimura, S. Masuda and T. Kosugi, Montana State University and the Institute od Space and Astronautical Science, 2003.

[21] M. A. Shea, D. F. Smart, and K. R. Pyle, "Direct solar neutrons detected by neutron monitors on 24 May 1990," Geophysical Research Letters, vol. 18, no. 9, pp. 1655-1658, 1991.

[22] M. A. Shea, D. F. Smart, and K. R. Pyle, in Proceedings of the 22nd International Cosmic Ray Conference (ICRC '91), vol. 3, pp. 57-60, Dublin, Ireland, 1991.

[23] G. E. Kocharov, L. G. Kocharov, G. A. Kovaltsov et al., in Proceedings of the 23rd International Cosmic Ray Conference (ICRC '93), vol. 3, pp. 107-110, Calgary, Canada, 1993.

[24] V. V. Akimov, N. G. Leikov, A. V. Belov et al., "Some evidences of prolonged particle acceleration in the high-energy gammaray flare of June 15, 1991," in Proceedings of the AIP Conference, J. Ryan and W. Vestrand, Eds., vol. 29 of High-Energy Solar Phenomena-A New Era of Spacecraft Measurements, pp. 106111, 1993.

[25] A. V. Belov and M.A. Livshits, "Neutron burst on may 24, 1990," Astronomy Letters, vol. 21, no. 1, pp. 37-40, 1995.

[26] D. F. Smart, M. A. Shea, and K. O'Brien, in Proceedings of the 24th International Cosmic Ray Conference (ICRC '95), vol. 4, pp. 171-174, Rome, Italy, 1995.

[27] H. Debrunner, J. A. Lockwood, C. Barat et al., "Energetic neutrons, protons, and gamma rays during the 1990 May 24 solar cosmic-ray event," The Astrophysical Journal, vol. 479, no. 2, pp. 997-1011, 1997.

[28] E. L. Chupp and J. M. Ryan, Research in Astronomy and Astrophysics, vol. 9, pp. 11-40, 2009.

[29] G. Rank, J. Ryan, H. Debrunner, M. McConnell, and V. Schonfelder, "Extended gamma-ray emission of the solar flares in June 1991," Astronomy \& Astrophysics, vol. 378, no. 3, pp. 1046-1066, 2001.

[30] K. R. Pyle and J. A. Simpson, in Proceedings of the 22nd International Cosmic Ray Conference (ICRC'91), vol. 3, pp. 5356, Dublin, Ireland, 1991.

[31] Y. Muraki, K. Murakami, M. Miyazaki et al., "Observation of solar neutrons associated with the large flare on 1991 June 4," Astrophysical Journal Letters, vol. 400, no. 2, pp. L75-L78, 1992.

[32] Y. Muraki, "New solar neutron detector and large solar-flare events of june 4th and 6th, 1991," Journal of Geomagnetism and Geoelectricity, vol. 47, no. 11, pp. 1073-1078, 1995.

[33] A. Struminsky, M. Matsuoka, and K. Takahashi, "Evidence of additional production of high-energy neutrons during the solar flare on 1991 June 4," Astrophysical Journal Letters, vol. 429, no. 1, pp. 400-405, 1994.

[34] R. A. Schwartz, B. R. Dennis, G. J. Fishman, C. A. Meegan, R. B. Wilson, and W. S. Paciesas, "BATSE flare observations in 
Solar Cycle 22," in Proceedings of the Compton Observatory Science Workshop, vol. 3137, pp. 457-468, NASA, 1992.

[35] R. J. Murphy, G. H. Share, and J. E. Grove, "OSSE observations of the 4 June 1991 solar flare," in Proceedings of the AIP Conference, J. Ryan and W. Vestrand, Eds., vol. 294, pp. 15-20, 1993.

[36] E. J. Scheneid, K. T. S. Brazier, and G. Kanbach, "EGRET observations of extended high-energy emissions from the nuclear line flares of June," in Proceedings of the AIP Conference, J. Ryan and W. Vestrand, Eds., vol. 294, pp. 94-99, 1993.

[37] S. Tsuneta and T. Naito, "Fermi acceleration at the fast shock in a solar flare and the impulsive loop-top hard x-ray source," Astrophysical Journal Letters, vol. 495, no. 1, pp. L67-L70, 1998.

[38] R. Ramaty and N. Mandzhaxvidze, "Theoretical models for high-energy solar flare emissions," in Proceedings of the AIP Conference, J. Ryan and W. Vestrand, Eds., vol. 294, pp. 26-44, 1993.

[39] N. Mandzhavidze and R. Ramaty, "High-energy gamma-ray emission from pion decay in a solar flare magnetic loop," Astrophysical Journal Letters, vol. 389, no. 2, pp. 739-755, 1992.

[40] H. E. Petschek, "Magnetic field annihilation," in Proceedings of the AAS-NASA Symposium, p. 425, Goddard Space Flight Center, 1964.

[41] S. Masuda, T. Kosugi, H. Hara, S. Tsuneta, and Y. Ogawara, "A loop-top hard X-ray source in a compact solar flare as evidence for magnetic reconnection," Nature, vol. 371, no. 6497, pp. 495-497, 1994.

[42] K. Shibata, "Evidence of magnetic reconnection in solar flares and a unified model of flares," Astrophysics and Space Science, vol. 264, pp. 129-144, 1999.

[43] T. Yokoyama and K. Shibata, "A two-dimensional magnetohydrodynamic simulation of chromospheric evaporation in a solar flare based on a magnetic reconnection model," The Astrophysical Journal, vol. 494, pp. L113-L116, 1998.

[44] G. D. Holman, "Particle acceleration in large-scale DC electric fields," in Proceedings of the AIP Conference, R. Ramaty and N. Mandzhavidze, Eds., vol. 206, pp. 135-144, 2000.

[45] T. Sako, K. Watanabe, Y. Muraki et al., "Long-lived solar neutron emission in comparison with electron-produced radiation in the 2005 September 7 solar flare," Astrophysical Journal Letters, vol. 651, no. 1, pp. L69-L72, 2006.

[46] Y. Muraki, in Proceedings of the 30th International Cosmic Ray Conference (ICRC '09), R. Caballero, J. C. D'Olivo, G. Medina-Tanco, and J. F. Valdés-Galicia, Eds., vol. 6, pp. 181194, Merida, Mexico, 2009.

[47] Y. T. Tanaka, IAU telegram \#3417 (for the flare of June 7, 2011) and \#3886 (for the flare of January 23, 2012). Paper presented at ICRC, Beijing, China, paper no. \#683, 2011.

[48] L. Dorman, Solar Neutrons and Related Phenomena, Astrophysics and Space Science Library Series 365, Springer, 2010.

[49] H. Matsumoto, T. Goka, K. Koga et al., "Real-time measurement of low-energy-range neutron spectra on board the space shuttle STS-89 (S/MM-8)," Radiation Measurements, vol. 33, pp. 321-333, 2001.

[50] T. Doke, "Radiation hazard for the astronauts who stay for long time in the space," Science Asahi, vol. 9, pp. 38-43, 1997.

[51] H. Koshiishi, H. Matsumoto, A. Chishiki, T. Goka, and T. Omodaka, "Evaluation of the neutron radiation environment inside the International Space Station based on the Bonner Ball Neutron Detector experiment," Radiation Measurements, vol. 42, no. 9, pp. 1510-1520, 2007.

[52] Hinode, http://solar-b.nao.ac.jp/index_e.shtml.
[53] Solar Dynamical Observatory, http://sdo.gsfc.nasa.gov/assets/. img/browse/2011/06/07/20110607_062513_1024_0171.jpg.

[54] N. Vilmer, A. L. MacKinnon, and G. J. Hurford, "Properties of energetic ions in the solar atmosphere from gamma-ray and neutron observations," Space Science Reviews, vol. 159, pp. 167-224, 2011.

[55] E. O. Flückiger, R. Bütikofer, A. Chlingalian et al., "Search for solar neutrons in association with large solar flares in July 2000 and March-April 2001," in Proceedings of the 27th International Cosmic Ray Conference (ICRC '01), pp. 3044-3047, 2001.

[56] Y. Muraki, H. Tsuchiya, K. Fujiki et al., "A solar neutron telescope in Tibet and its capability examined by the 1998 November 28th event," Astroparticle Physics, vol. 28, no. 1, pp. 119-131, 2007.

[57] J. F. Valdes-Galicia, Y. Muraki, H. Tsujihara et al., "An improved solar neutron telescope installed at a very high altitude in Mexico," Nuclear Instruments and Methods in Physics Research A, vol. 535, pp. 656-664, 2004.

[58] L. X. González, F. Sánchez, and J. F. Valdés-Galicia, “Geant4 simulation of the solar neutron telescope at Sierra Negra, Mexico," Nuclear Instruments and Methods in Physics Research A, vol. 613, pp. 263-271, 2010.

[59] K. Koga, T. Goka, H. Matsumoto, Y. Muraki, K. Masuda, and Y. Matsubara, "Development of the fiber neutron monitor for the energy range $15-100 \mathrm{MeV}$ on the International Space Station (ISS)," Radiation Measurements, vol. 33, no. 3, pp. 287291, 2001.

[60] K. Koga, T. Goka, H. Matsumoto, and Y. Muraki, in Proceedings of the 31st International Cosmic Ray Conference (ICRC '09), vol. 831, Lodz, Poland, 2009.

[61] K. Koga, T. Goka, H. Matsumoto, T. Obara, Y. Muraki, and T. Yamamoto, in Proceedings of the 21st ECRS, K. Kudela, P. Kiraly, and A. Wolfendale, Eds., pp. 199-200, Kosice, Slovakia, 2008.

[62] K. Koga, T. Goka, H. Matsumoto, T. Obara, Y. Muraki, and T. Yamamoto, "Measurement of high-energy neutrons at ISS by SEDA-AP," Astrophysics and Space Sciences Transactions, vol. 7, pp. 411-416, 2011.

[63] I. Imaida, Y. Muraki, Y. Matsubara et al., "A new tracking satellite-borne solar neutron detector," Nuclear Instruments and Methods in Physics Research A, vol. 421, no. 1-2, pp. 99-112, 1999.

[64] K. Watanabe, in Proceedings of the Cosmic-Ray Research of Nagoya University, vol. 46, pp. 1-249, 2005.

[65] Y. Muraki, Y. Matsubara, S. Masuda et al., "Detection of highenergy solar neutrons and protons by ground level detectors on April 15, 2001," Astroparticle Physics, vol. 29, no. 4, pp. 229242, 2008.

[66] S. N. Kuznetsov, V. G. Kurt, I. N. Myagkova, B. Y. Yushkov, and K. Kudela, "Gamma-ray emission and neutrons from solar flares recorded by the SONG instrument in 2001-2004," Solar System Research, vol. 40, no. 2, pp. 104-110, 2006.

[67] D. Shiota, S. Tsuneta, M. Shimojo, N. Sakao, D. Orozco Suárez, and R. Ishikawa, "Polar field reversal observations with Hinode," The Astrophysical Journal, vol. 753, p. 157, 2012.

[68] Y. Hanaoka, "Double-loop configuration of solar flares," Solar Physics, vol. 173, no. 2, pp. 319-346, 1997. 

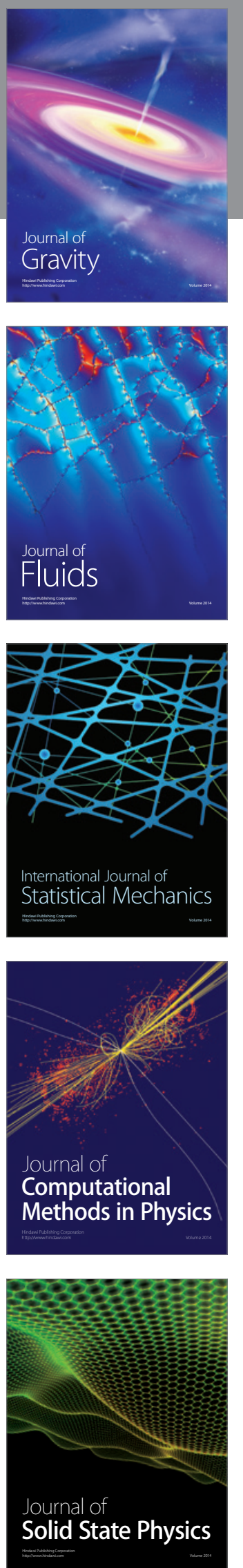

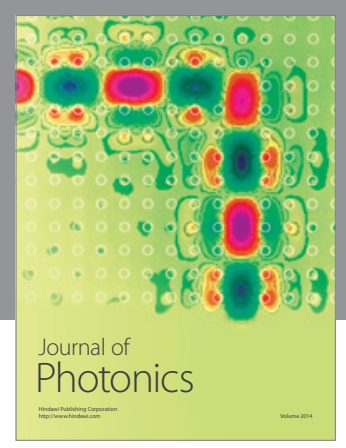

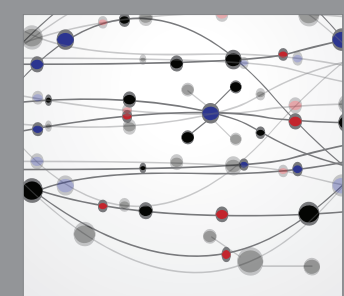

The Scientific World Journal
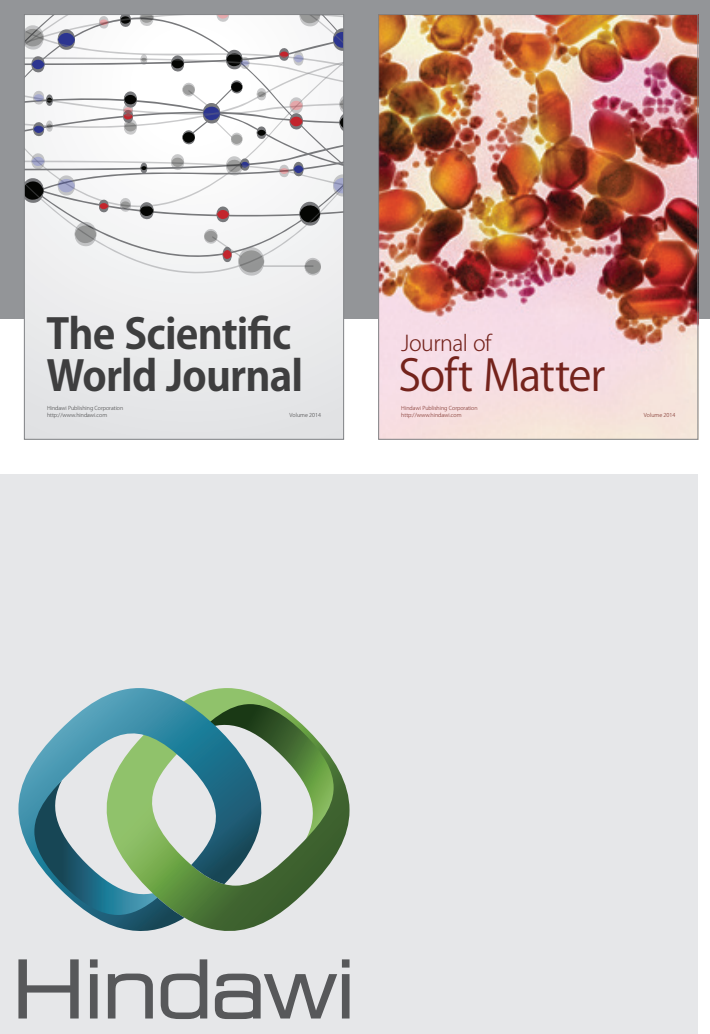

Submit your manuscripts at

http://www.hindawi.com
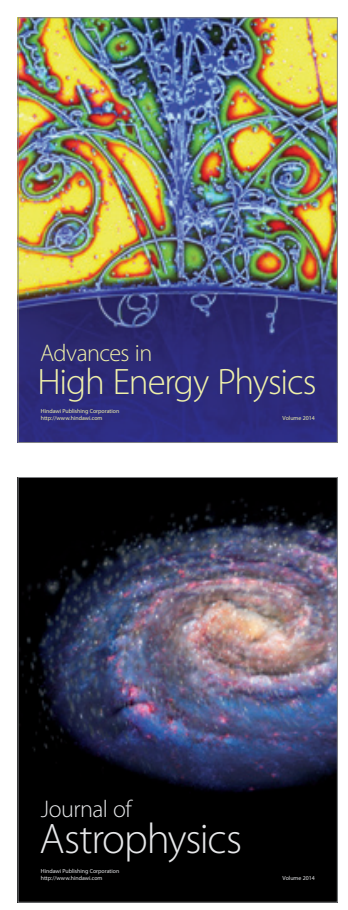
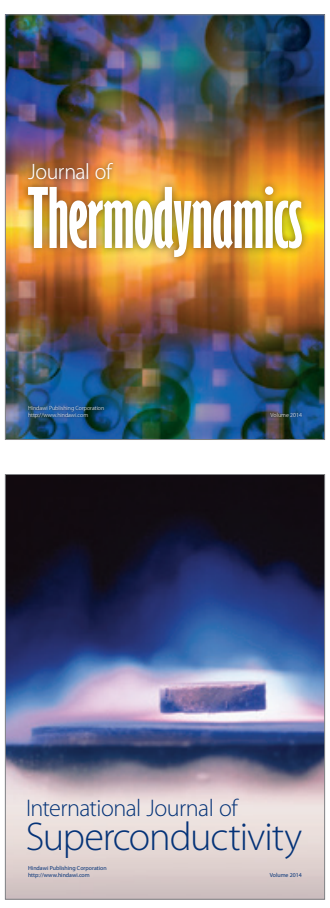
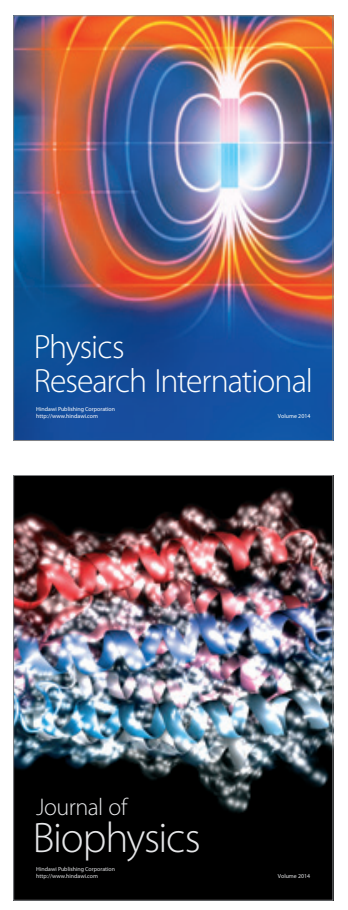
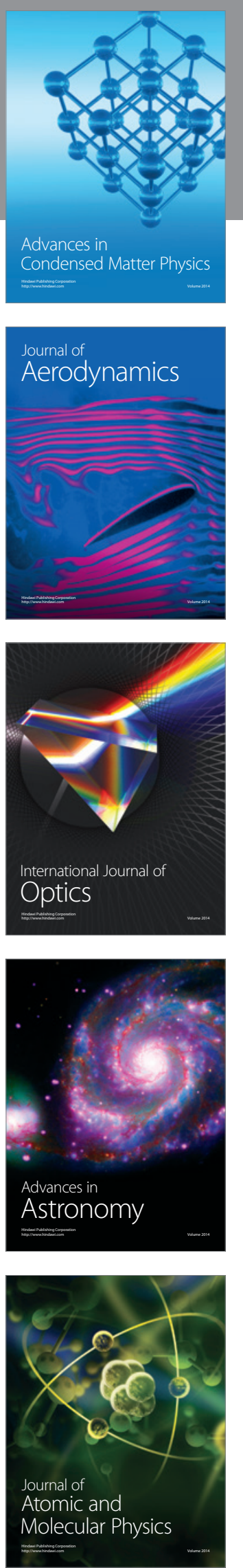